\title{
Nanoelectromechanical Systems: A New Opportunity For Microelectronics
}

\author{
Michael ROUKES \\ Co-Director, Kavli Nanoscience Institute (http://kni.caltech.edu) \\ Professor of Physics, Applied Physics, and Bioengineering, California Institute of Technology \\ Co-Founder/Science and Technology Director \\ The Alliance for Nanosystems VLSI (www.nanovlsi.com), Grenoble, France \\ (roukes@caltech.edu)
}

\begin{abstract}
Transitioning nanoscale devices from the realm of one-of-a-kind feats into robust and reproducible nanosystems - that is, useable technology - is a monumental challenge that transcends the capabilities of any one laboratory. Some solid progress is now being achieved toward this end, and these efforts are critical for realizing the promise of "active" nanotechnology. At least two essential elements must be in place to realize the vast applications potential that awaits. First, an unfamiliar fusion of technologies is required; one that melds
\end{abstract}

techniques from traditionally-separate disciplines with an appropriate scale of approach. Second, robust methods for large-scale-integration of complex nanodevices are required, and these must engender routes to production en masse. To illustrate the emerging potential of nanosystems I will describe several advances in nanoelectromechanical systems (NEMS) that offer immense promise for applications ranging from metrology, environmental sensing, the life sciences, and health care. 